Check for updates

Cite this: RSC Adv., 2017, 7, 37268

\title{
Construction of chain segment structure models, and effects on the initial stage of the thermal degradation of poly(vinyl chloride) $\uparrow$
}

\author{
Yin Lv, ${ }^{\text {ab }}$ Jia Liu, ${ }^{a}$ Zhidong Luo, ${ }^{a}$ Heyun Wang ${ }^{a}$ and Zhong Wei (DD *a
}

The thermal degradation of poly(vinyl chloride) (PVC) is inevitable during its processing and is directly related to the initial stage of the thermal stability of PVC. This paper focuses on the influence of the segment structures that include a conjugated polyene sequence structure and internal allylic chloride structure on the initial stage of the thermal degradation of PVC. The conjugated polyene sequence structure and internal allylic chloride structure were the structural models successfully constructed via thermal and alkali treatment methods through the long chain of PVC directly. Ultraviolet-visible spectroscopy (UV-Vis) was used to analyze the type and content of the conjugated polyene structure. Nuclear magnetic resonance spectroscopy $\left({ }^{1} \mathrm{H}\right.$-NMR) was used to determine the variation trend in the content of internal allylic chlorine structures because of the oxygen-containing structure excluded by Fourier-transform infrared spectrometer (FTIR) characterization. The thermal stability of the treated PVC was also determined through thermogravimetric analysis (TGA). The comparison between the two structural models revealed that the internal allylic chloride structure greatly influenced the initial thermal stability as an initiation point in the PVC chain, thereby leading to thermal instability.

Received 11th July 2017

Accepted 21st July 2017

DOI: $10.1039 / \mathrm{c} 7 \mathrm{ra0} 07615 \mathrm{k}$

rsc.li/rsc-advances

tertiary chloride, double bond along the direction of the main

\section{Introduction}

Poly(vinyl chloride) (PVC) is one of the most widely used thermoplastics because of its various applications. However, problems in its degradation and stability during manufacturing remain and have attracted extensive research attention. ${ }^{1-8}$ PVC is molecularly unstable toward heat, and this instability is much more pronounced for PVC than for its major competitors. ${ }^{\mathbf{9} 10}$ Usually, PVC can form an irregular molecular chain structure (defect structure) under PVC polymerization conditions, processing technology, and post-treatment, which had affected the thermal stability of PVC. Furthermore, defects can result from several different mechanisms, some of which have not yet been finalized, and any kind of defect can lead to poor thermal stability of PVC. This thermal stability of resin can limit the machining performance and scope of use of PVC. Defect structures include head-head structure in the process of chain transfer, end of the unsaturated group, chlorine methyl group,

${ }^{a}$ Key Laboratory for Green Processing of Chemical Engineering of Xinjiang Bingtuan, School of Chemistry and Chemical Engineering, Shihezi University, Shihezi 832000 , China.E-mail: steven_weiz@sina.com; ag_125@163.com; liujia@stu.shzu.edu.cn; luodong.zhi@163.com; 18609932991@163.com

${ }^{b}$ Key Laboratory of Materials-Oriented Chemical Engineering of Xinjiang Uygur Autonomous Region, Engineering Research Center of Materials-Oriented Chemical Engineering of Xinjiang Bingtuan, School of Chemistry and Chemical Engineering, Shihezi University, Shihezi, China

$\dagger$ Electronic supplementary information (ESI) available. See DOI: $10.1039 / \mathrm{c} 7 \mathrm{ra} 07615 \mathrm{k}$ carbon chain, allyl group, carbonyl group, and terminal groups resulting from the initiator (e.g., peroxide or hydroperoxide group). In the above-mentioned defect structure, tertiary chloride is one of important factors affecting the thermal stability of PVC. ${ }^{18}$ In addition, allyl chloride and conjugated polyene defect structures are formed by polymerization and are intermediates in the thermal degradation of PVC. These structures increase with the hot-working character and degradation behavior of PVC and are thus attracting increased attention.

In all cases, the degradation of the primary chain of the PVC is caused by the zip elimination of $\mathrm{HCl}$ at low temperatures, ${ }^{\mathbf{1 1 - 1 4}}$ as well as by the inevitable formation of the conjugated polyene sequence structure and allylic chloride structure in the polymer chain. All these factors affect the processing and use of PVC, but most studies ${ }^{12,15-18}$ have focused on the initial dehydrochlorination of PVC.

Some studies ${ }^{19,20}$ have considered that the initial stage of PVC thermal degradation is initiated by the decomposition of normal units and by a series of intermediates. These initiators lead to a conjugated polyene sequence structure and internal chloroallylic fragments, which have been proposed by mathematical models or small-molecule-compound models.

A number of studies ${ }^{21-24}$ have shown that $\mathrm{HCl}$ loss occurs at defects and at normal units of the polymer. This chemical process regulates the relatively large concentrations of internal allylic chlorines, together with the same concentration of short conjugated double-bond sequence. Other studies ${ }^{23,29}$ have also 
proposed to combine determinate results based on ozonolysis with GPC characterization for different polymerized samples, which does not take into account the double bond located near the chain end and makes no difference between a polyene sequence and an isolated double bond (one chain scission in each case). ${ }^{23,25}$ To obtain double bonds in PVC, copolymerizations of vinyl chloride (VC) with phenylacetylene (PA) and dimethylester of 2-butyne dioic acid (ADCE) have been performed. Braun ${ }^{15}$ assumed that the accelerating effect of conjugated phenyl groups greatly exceeds the effect of olefinic double bonds on the $\mathrm{HCl}$ evolution rate. However, the small-moleculecompound model is insufficient to provide it directly.

Abbås ${ }^{26}$ showed that dehydrochlorination is predominantly initiated at internal unsaturation structure (allylic chloride) sites. Compared with an intact vinyl chloride unit, the $\mathrm{Cl}-\mathrm{C}$ bond in the chloroallyl structure is less stable, so the elimination of $\mathrm{HCl}$ likely occurs in such a structure. ${ }^{20,27,28}$ The double bonds inside the chain reduces thermal stability far more markedly than they do at the end of the chain. ${ }^{29}$ However, whether the conjugated polyene sequence structure and allylic chloride structure play an important role in the initial stage of PVC thermal degradation remains unclear.

Studies on the initial thermal stability and thermal degradation of PVC are abundant, and these processes remain difficult to elucidate. The reason is that they involve complex-chain dehydrochlorination that undergoes an initiation process to generate an active intermediate and a series of chain reactions that produce additional active intermediates with progressively increasing number of double bonds. ${ }^{30-32}$ The conjugated polyene sequence structure and the allylic chloride structure play an important role in the initial stage of the thermal degradation of PVC. However, investigations on these structures are inconsistent and controversial. Meanwhile, few works have been conducted on the systematic classification of the complicated construction at the initial stage of PVC thermal stability. Most researchers have adopted mathematical models or smallmolecule-compound models to investigate the initial stage of PVC thermal degradation. Unfortunately, these models cannot sufficiently explain the relationship between the structures and properties of PVC.

Given this problem, many different perspectives have been presented regarding the initial stage of the thermal degradation of PVC. Studying the factors influencing the initial thermal dehydrochlorination process and providing direct evidence on the thermal degradation of PVC through experimentation is indispensable.

On the basis of our previous studies, ${ }^{33}$ we have constructed in the present work the conjugated polyene sequence structure model (CSM) and the allylic chlorine structure model (ASM) through PVC chains directly by thermal pretreatment and alkali pretreatment, respectively. We aimed to explore the initial dehydrochlorination process of PVC resin. For the CSM, in the process of building the polyene structure model by thermal treatment, the independent internal allyl chloride structure similar to defects on the molecular chain was the starting point. Next was conforming the zip elimination of $\mathrm{HCl}$, which in turn generated a conjugated diene, conjugated triene, etc. In addition, considering the catalysis on $\mathrm{HCl}$ elimination by alkali, ASM can form an independent internal double bond and then generate the conjugated polyene sequence by $\mathrm{HCl}$ elimination. Therefore, increasing the content of conjugated polyene structure greatly improved the content of allyl chloride.

Direct and objective experimental data were obtained to establish the relationship of various subdivision structures with the thermal degradation property of PVC. The main initial degradation structures were examined by ultraviolet-visible spectroscopy (UV-Vis), Fourier transform infrared spectrometry (FTIR), and nuclear magnetic resonance spectrometry $\left({ }^{1} \mathrm{H}\right.$ NMR). The thermal performance of PVC was examined by thermogravimetric analysis (TGA). Then the effects of conjugated polyene structures and internal allylic chloride structures on the thermal stability of PVC had been compared.

\section{Experimental}

\subsection{Materials}

The polymerized PVC resin suspension was purchased from Xinjiang Tianye Chemical Plant (Xinjiang, China). Tetrahydrofuran (THF) and 1,2-dichloroethane (DCE) were supplied by Sinopharm Chemical Reagent Co., Ltd. Methanol and ethanol were obtained from Fuyu Chemical Corporation (Tianjin, China). Sodium hydroxide was provided by Shengao chemical reagent (Tianjin) Co., Ltd. All chemical solvents were of analytical grade and used without further treatment.

\subsection{Preparation and pretreatment of PVC samples}

The crude PVC was dissolved in THF to obtain purified PVC samples, which were then precipitated with methanol to remove the additives, such as stabilizers and plasticizers. ${ }^{34}$

(a) Thermal aging tests were conducted in a customized chamber equipment. The heating tests were conducted up to 40 days (total time) under $120{ }^{\circ} \mathrm{C}$ at a flow rate of $100 \mathrm{~mL} \mathrm{~min}{ }^{-1}$ under nitrogen atmosphere. The samples were collected within a certain period of time.

(b) The alkali treatment methods were as follows. In a 500 $\mathrm{mL}$ three-necked flask equipped with a stirrer, a thermometer, and a reflux condenser, a mixture of $10 \mathrm{~g}$ of purified PVC and $200 \mathrm{~mL}$ of THF were stirred at $4{ }^{\circ} \mathrm{C}$ until a uniform solution was obtained. A weighed amount of $\mathrm{NaOH}$ dissolved in $50 \mathrm{~mL}$ of EtOH was then added to the resultant solution. Every once for a certain time, a specific volume of solution was taken out and poured into a large amount of water. The resulting precipitates were washed several times with distilled water. Then the precipitates were dissolved in approximately $100 \mathrm{~mL}$ of 1,2dichloroethane and washed with distilled water to $\mathrm{pH}$ neutral. The resulting solution was added with $300 \mathrm{~mL}$ of methanol. The precipitates were collected and then dried in a vacuum oven at $30{ }^{\circ} \mathrm{C}$ to constant weight.

\subsection{Characterization}

The molecular weight distributions (MWD) and the average molecular weights were determined using Waters 2695 Alliance Gel Permeation Chromatography (Waters, USA) equipped with 
PL-Gel columns. All measurements were obtained at $40{ }^{\circ} \mathrm{C}$ in THF with narrow MWD polystyrene as standard. A calibration curve was obtained with the following seven polystyrene standards: 1200, 3070, 7210, 19 600, 49 200, 113 000, and 257000 Da of low polydispersity.

The UV absorption spectra of the PVC solutions were recorded at room temperature, using air as reference and DCE. ${ }^{35}$ The spectrum at 200-600 nm was obtained by using a V-1100D UVvisible spectrophotometer (Shanghai, China).

${ }^{1} \mathrm{H}-\mathrm{NMR}$ spectra was obtained by using a $400 \mathrm{MHz}$ Bruker Avance III HD spectrometer. The samples for the ${ }^{1} \mathrm{H}-\mathrm{NMR}$ experiments were prepared by using a $5 \% \mathrm{w} / \mathrm{v}$ solution of PVC in THF- $\mathrm{d}_{8}$. Pre-acquisition delays were optimized, and the digital signal processing was used to obtain a flat baseline for accurate integration.

The infrared spectra of the samples were obtained by using an ISIO FTIR spectrometer (Thermo Fisher Scientific, USA).

NETZCH model STA449F3 Jupiter (NETZCH, Germany) was used to determine the thermal stability of the sample. Dynamic and static analyses were employed. The temperature when the weight loss of samples was $5 \%\left(T_{5 \%}\right)$ was used to evaluate the initial thermal stability of the samples. The dynamic measurement conditions were as follows: approximately $5.2 \mathrm{mg}$ of sample was used under nitrogen atmosphere at a flow rate of $100 \mathrm{~mL} \mathrm{~min}^{-1}$ and heating rate of $10{ }^{\circ} \mathrm{C} \mathrm{min}^{-1}$. The static measurement conditions were as follows: isothermal analyses at $120{ }^{\circ} \mathrm{C}$ for $2 \mathrm{~h}$ was performed using $5.2 \mathrm{mg}$ of samples under nitrogen atmosphere at a flow rate of $100 \mathrm{~mL} \mathrm{~min}^{-1}$. The set temperature was attained at a heating rate of $10{ }^{\circ} \mathrm{C} \mathrm{min}^{-1}$.

\section{Results and discussion}

Several researchers ${ }^{28,30,36,37}$ have proposed that the degradation of PVC resin under inert atmosphere is mainly caused by dehydrochlorination. Thus, we measured the number-average $\left(M_{\mathrm{n}}\right)$ and weight-average $\left(M_{\mathrm{w}}\right)$ molecular weights of PVC resins, as well as their polydispersity indices (Appendices, Table A. $1^{\dagger}$ ). With the extension of alkali and thermal pretreatment times, all polymers exhibited similar molecular weight and molecular-weight distribution values, and no insoluble substances were observed. Therefore, dehydrochlorination occurred because of the alkali and thermal treatments, and no chain scission and crosslinking reactions occurred.

\subsection{Thermal pretreatment and construction of CSM}

To reveal the relationship between the segment structures and the thermal properties of PVC, preheating treatment method was used to construct the PVC chain segment model. The PVC resins were treated at $120^{\circ} \mathrm{C}$ in nitrogen environment at varying periods to construct the CSM. The reaction to remove $\mathrm{HCl}$ from the PVC chain predominantly involved the production of conjugated polyene sequences, and all other reactions were negligible when the PVC samples were heated at $<200{ }^{\circ} \mathrm{C}$ in nitrogen atmosphere. ${ }^{38-40}$ Moreover, a minimal amount of allylic chloride structures in the PVC main chain was introduced through resin polymerization.

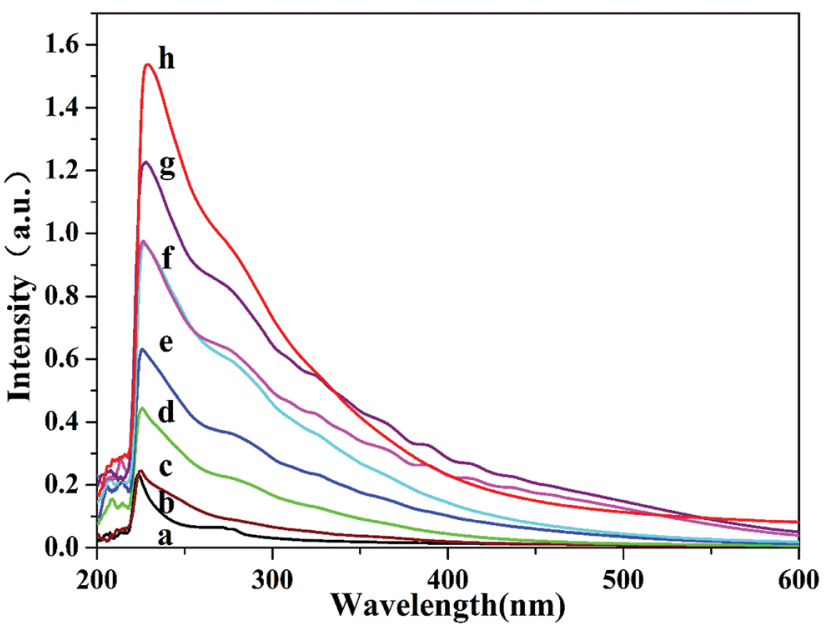

Fig. 1 Absorption spectra of PVC under different thermal pretreatment time (a-0 d, b-1 d, c-3 d, d-5 d, e-7 d, f-8 d, g-9 d, and h-10 d).

Based on our research work, ${ }^{35}$ UV-Vis characterization was designed to investigate the types of conjugated polyene sequence structure in commercial PVC resins. Fig. 1 shows that absorption increased as a function of the preheat treatment time, suggesting that the conjugated polyene sequence structure was generated. Based on the conjugated polyene sequences using UV-Vis, the data and quantitative calculation results of the polyene structures are listed in Table 1, which shows that the content of conjugated polyene sequences increased over the preheat treatment time.

To rule out the introduction of oxygen in the processes, FTIR analysis can facilitate the periodic measurement of functionalgroup variation. The corresponding changes in the structures of the samples are shown in the Appendices (Fig. A.1†). In the FTIR spectra, the wavenumber of $1427 \mathrm{~cm}^{-1}$ corresponded to the bending vibration absorption peak of $-\mathrm{CH}_{2}$ - in the PVC chain. The lack of signals from the FTIR absorption peaks at 1700$1800 \mathrm{~cm}^{-1}$ and $3300-3500 \mathrm{~cm}^{-1}$ indicated that no oxygencontaining structures were formed in the pretreatment process.

To determine the types and contents of segment structures in the PVC main chain, ${ }^{1} \mathrm{H}-\mathrm{NMR}^{20,41-44}$ was used to analyze the

Table 1 Content of conjugated polyene sequences under different heat treatment times

Content/1000 vinyl chloride structure units

Number of conjugated double bond $n$

\begin{tabular}{lllll}
\hline Samples & 2 & 3 & 4 & 5 \\
\hline a-0 d & 0.1326 & 0.02969 & 0.009084 & 0.005056 \\
b-1 d & 0.1275 & 0.03526 & 0.01058 & 0.00580 \\
c-3 d & 0.2192 & 0.08130 & 0.02623 & 0.01452 \\
d-5 d & 0.3119 & 0.1328 & 0.0456 & 0.0261 \\
e-7 d & 0.3960 & 0.2054 & 0.0684 & 0.0383 \\
f-8 d & 0.4902 & 0.2331 & 0.08172 & 0.04748 \\
g-9 d & 0.5261 & 0.3546 & 0.1161 & 0.0637 \\
h-10 d & 0.5825 & 0.3072 & 0.1061 & 0.06120
\end{tabular}



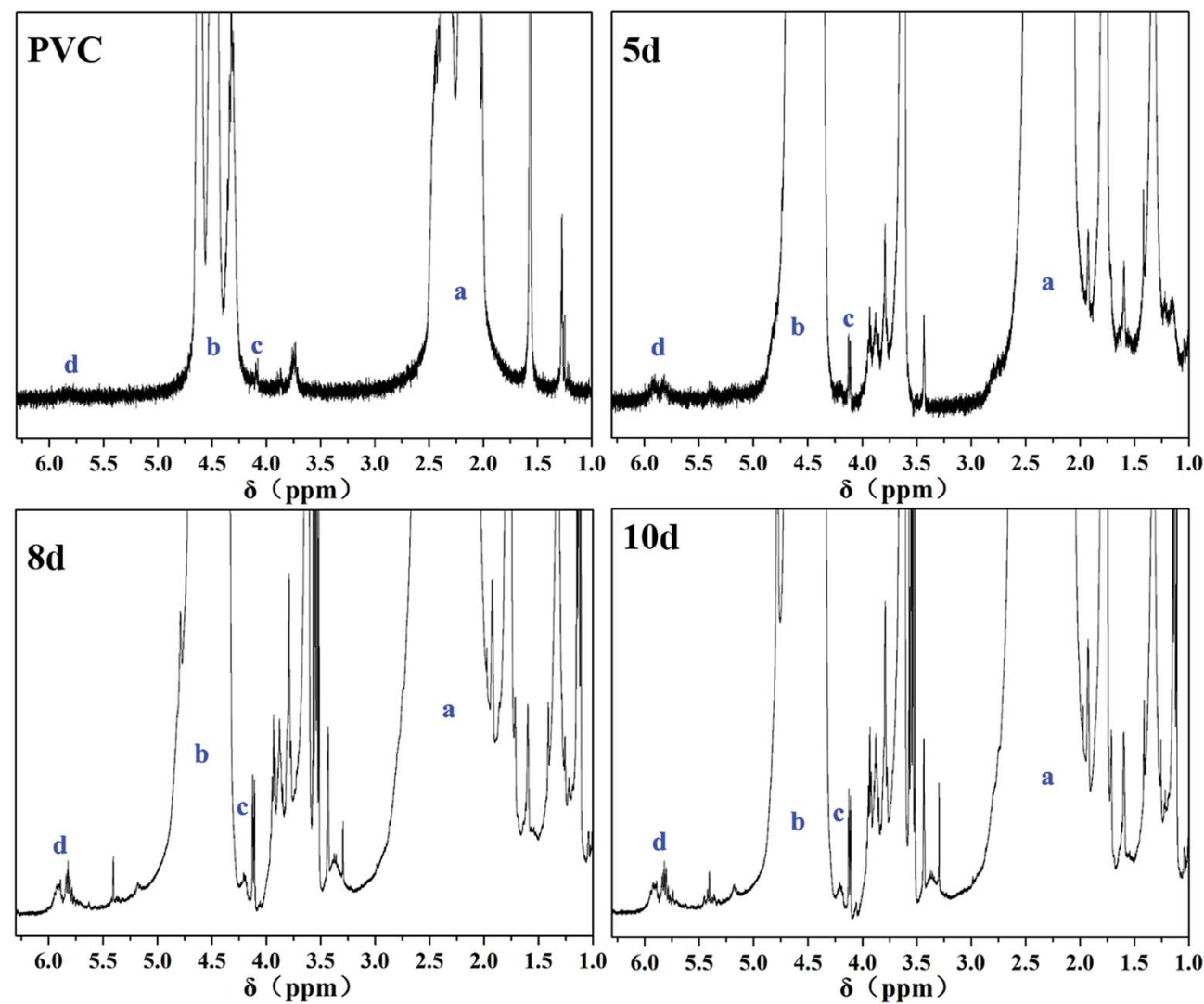

Fig. $2{ }^{1} \mathrm{H}$-NMR spectra of PVC under different thermal treatment times.

variation in PVC chain segment. In the ${ }^{1} \mathrm{H}-\mathrm{NMR}$ spectra, the intensity of -CHCl- (4.16-5.1 ppm, b, Fig. 2) in the PVC chain was used as the internal standard. If $N_{\mathrm{T}}$ is the average number of total double bonds (5.1-6.0 ppm, d, Fig. 2) and $N_{\mathrm{t}}$ is the average number of pseudo terminal double bonds (4.0$4.16 \mathrm{ppm}$, a, Fig. 2) per $1000 \mathrm{VC}$ monomer units, $N_{\mathrm{T}}-N_{\mathrm{t}}$ is the average number of internal double bonds per $1000 \mathrm{VC}$ monomer units $\left(N_{\mathrm{i}}\right)$, and the content of the internal allylic chlorines is similar to $N_{\mathrm{i}}$. Results are shown in Table 2.

Table 2 shows that the content of the internal allylic chloride structure of samples pretreated by thermal method underwent no apparent changes within a certain scope, indicating that the CSM model was successfully constructed.

Furthermore, to reveal the relationship between the conjugated polyene sequences structure and the thermal properties of PVC, curves from the pyrolysis of PVC pretreated by thermal method were obtained and are shown in Fig. 3. From the weight-loss curves, $T_{5 \%}$ was obtained, as shown in the Table 3 . These characteristic parameters depended on the sample properties and revealed the initial thermal stability of the samples. Fig. 3 shows no change with pretreatment time. $T_{5 \%}$ obtained from the TGA of the thermal pretreatment samples had no apparent variation within a certain time range. The conjugated polyene sequence structures did not evidently influence the initial thermal stability of resin, and no initiation sites were induced in the PVC main chain.

\subsection{Alkali pretreatment and construction of ASM}

Several studies ${ }^{20,27-29}$ have suggested that the internal allylic chloride in the PVC main chains significantly affect the thermal stability of PVC. To reveal the relationship between the segment structures and thermal properties of PVC, alkali-treatment

Table 2 Structure types and content in the PVC molecular chain under different thermal pretreatment times

\begin{tabular}{|c|c|c|c|c|c|}
\hline \multirow[b]{2}{*}{ Number } & \multirow[b]{2}{*}{ Structure types } & \multirow[b]{2}{*}{ Chemical shift $\delta / \mathrm{ppm}$} & \multicolumn{3}{|c|}{ Content/1000 VC } \\
\hline & & & $d-5 d$ & $f-8 d$ & $h-10 d$ \\
\hline $\mathrm{b}$ & $-\mathrm{CH}^{*} \mathrm{Cl}-$ & $4.16-5.1 \mathrm{ppm}$ & 1000 & 1000 & 1000 \\
\hline $\mathrm{c}$ & $-\mathrm{CH}=\mathrm{CH}-\mathrm{CH}_{2}^{*} \mathrm{Cl}$ & $4.10-4.16 \mathrm{ppm}$ & 0.7 & 1.5 & 1.1 \\
\hline $\mathrm{d}$ & $-\mathrm{CH}=\mathrm{CH}-($ total $)$ & 5.1-6.1 ppm & 4.2 & 3.7 & 3.6 \\
\hline- & $-\mathrm{CH}=\mathrm{CH}-$ (internal) & $5.1-6.1 \mathrm{ppm}$ & 3.5 & 2.2 & 2.5 \\
\hline
\end{tabular}




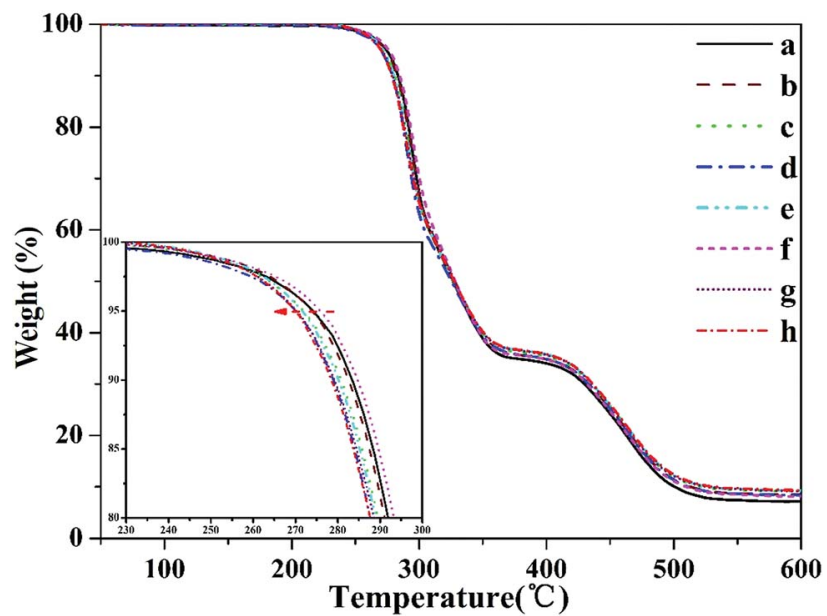

Fig. 3 TGA curves of PVC under different heat pretreatment times (a$0 d, b-1 d, c-3 d, d-5 d, e-7 d, f-8 d, g-9 d$, and h-10 d).

method was used to construct the ASM involving double bonds and conjugated polyene sequence structures. Fig. 4 shows that the content of conjugated polyene sequence structures increased with alkali-treatment time. The data and quantitative calculation results for the polyene structures are listed in Table 4, which shows that the content of the conjugated polyene sequences increased over alkali-treatment time. The oxygencontaining structure was not induced in the PVC main chains in the pretreatment process, as shown in the Appendices (Fig. A. $2 \dagger$ ).

However, the content of internal allylic chloride structure in the PVC main chain of samples pretreated through alkali method increased with time, as calculated from Fig. 5. Results are shown in Table 5. These variations indicated that the vinyl chlorides were randomly attacked by $\mathrm{OH}^{-}$, leading to the formation of the internal allylic chloride structure and then to the generation of conjugated polyene sequence structure accompanied by dehydrochlorination. These results proved that ASM was constructed.

To determine the thermal properties of PVC under alkali pretreatment, curves from the pyrolysis of PVC subjected to thermal pretreatment were obtained and are as shown in Fig. 6. Based on the weight-loss curves, $T_{5 \%}$ was obtained (Table 6). Fig. 6 shows that samples pretreated with alkali for different times were more unstable. Moreover, $T_{5 \%}$ obtained through TGA as a function of time apparently decreased, as indicated in Table 6.

The above analysis indicated that the initial thermal stability of PVC was greatly affected by the internal allylic chloride structure. However, the content of conjugated

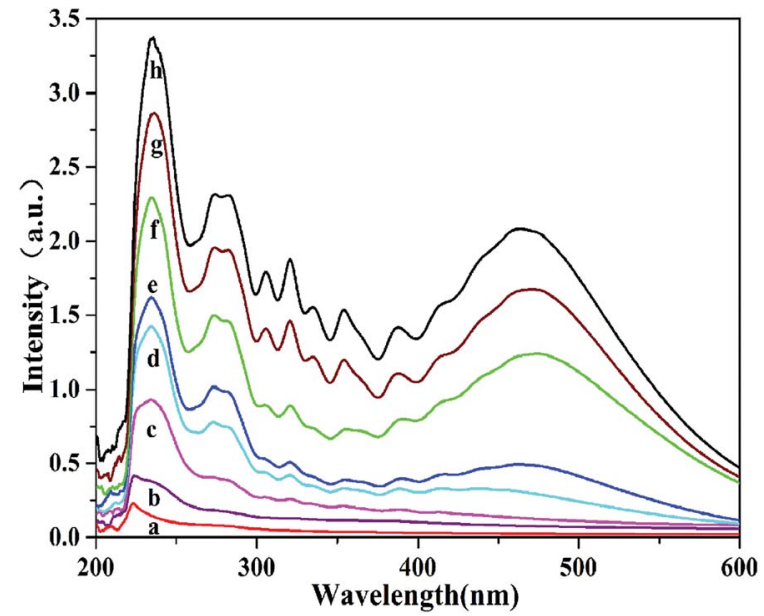

Fig. 4 Absorption spectra of PVC under different alkali treatment time (a-0 h, b-1 h, c-3 h, d-5 h, e-8 h, f-10 h, g-12 h, h-13.5 h).

Table 4 Content of conjugated polyene sequences under different alkali treatment times

\begin{tabular}{lllll}
\hline & \multicolumn{4}{l}{ Content/1000 vinyl chloride structure units } \\
\cline { 2 - 5 } & \multicolumn{4}{l}{ Number of conjugated double bond $n$} \\
\cline { 2 - 5 } Samples & 2 & 3 & 4 & 5 \\
\hline a-0 h & 0.1326 & 0.02969 & 0.009084 & 0.005056 \\
b-1 h & 0.2407 & 0.06770 & 0.02269 & 0.01377 \\
c-3 h & 0.4736 & 0.1473 & 0.04631 & 0.02749 \\
d-5 h & 0.6084 & 0.2636 & 0.07505 & 0.04393 \\
e-8 h & 0.6866 & 0.3363 & 0.09064 & 0.05299 \\
f-10 h & 0.8699 & 0.4944 & 0.1522 & 0.09186 \\
g-12 h & 0.9934 & 0.6448 & 0.2385 & 0.1477 \\
h-13.5 h & 1.1395 & 0.7610 & 0.3022 & 0.1891
\end{tabular}

polyene sequence structure obtained through alkali method was higher than that of samples pretreated with heat. Thus, we compared the results obtained through alkali pretreatment at $3 \mathrm{~h}$ (Table 4, sample c-3 h, 0.4736-0.01040) with those obtained through thermal pretreatment at 8 days (Table 1 , sample $f-8$ d, 0.4902-0.01357). Results showed that the contents of conjugated polyene sequence structure of the two samples were similar, and the former was lower than the latter. Comparison of thermal and alkali pretreatments showed that $T_{5 \%}$ decreased by about $10^{\circ} \mathrm{C}$ (Table 3, sample f- 8 d, $275.8{ }^{\circ} \mathrm{C}$; Table 6 , sample c-3 h, $265.6{ }^{\circ} \mathrm{C}$ ). The conjugated polyene sequence structure did not influence the initial thermal stability of PVC. Meanwhile, comparing the results obtained through alkali pretreatment at $1 \mathrm{~h}$ (Table 4, sample

Table 3 Thermal decomposition results of PVC as a function of thermal pretreatment times

\begin{tabular}{llllllll}
$\begin{array}{l}\text { Thermal pretreatment } \\
\text { time (d) }\end{array}$ & a-0 d & b-1 d & c-3 d & d-5 d & e-7 d & f-8 d & g-9 d \\
\hline$T_{5 \%}\left({ }^{\circ} \mathrm{C}\right)$ & 274.8 & 274.0 & 272.0 & 275.0 & 271.7 & 275.8 & 270.3
\end{tabular}



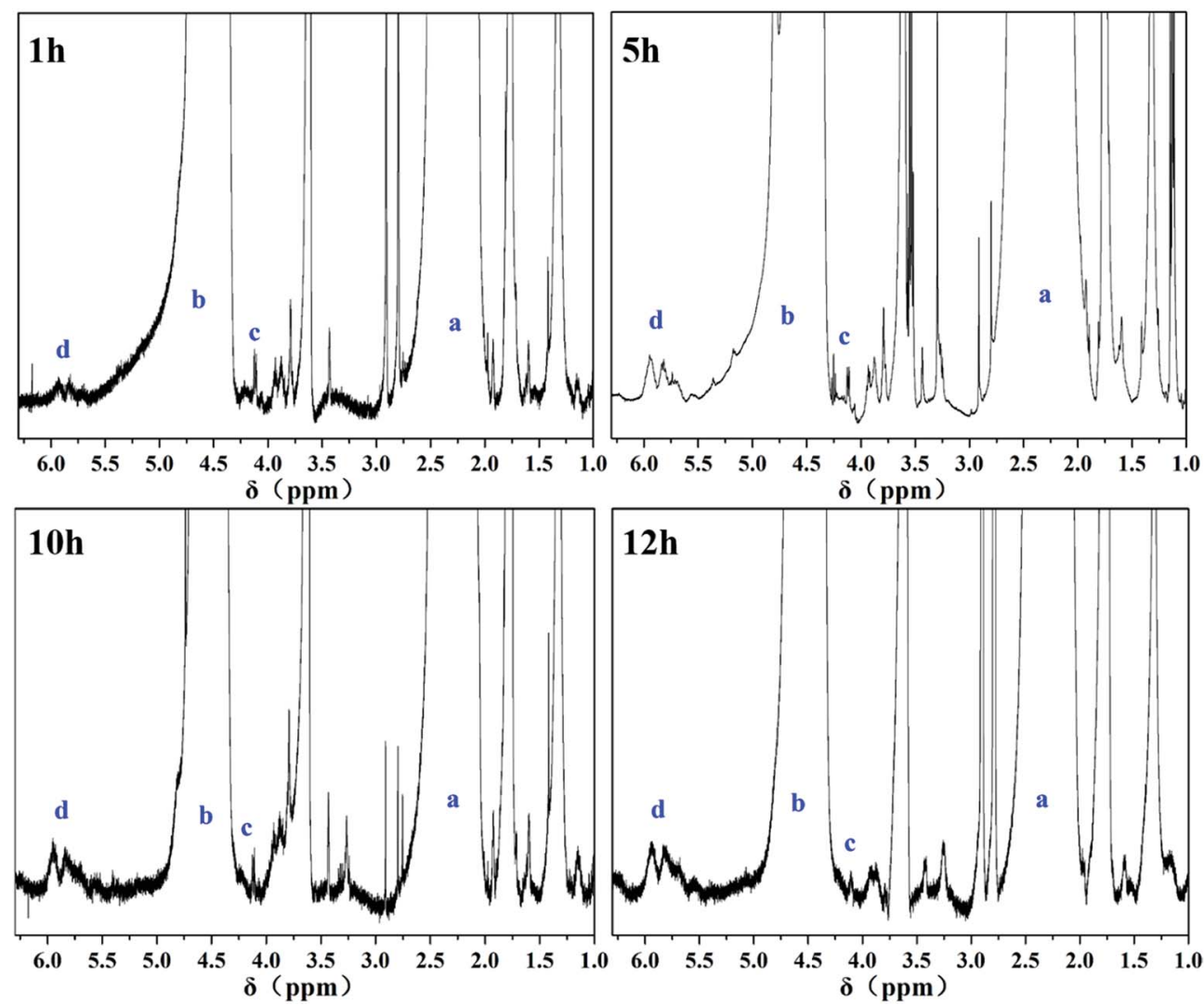

Fig. $5{ }^{1} \mathrm{H}$-NMR spectra of PVC under different chemical treatment times.

Table 5 Structure types and content of the molecular chain in the PVC resin under different chemical treatment times

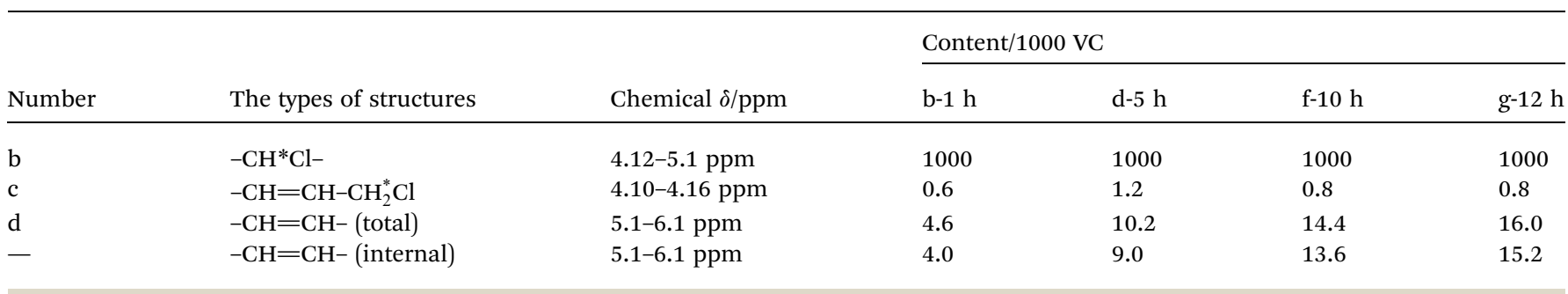

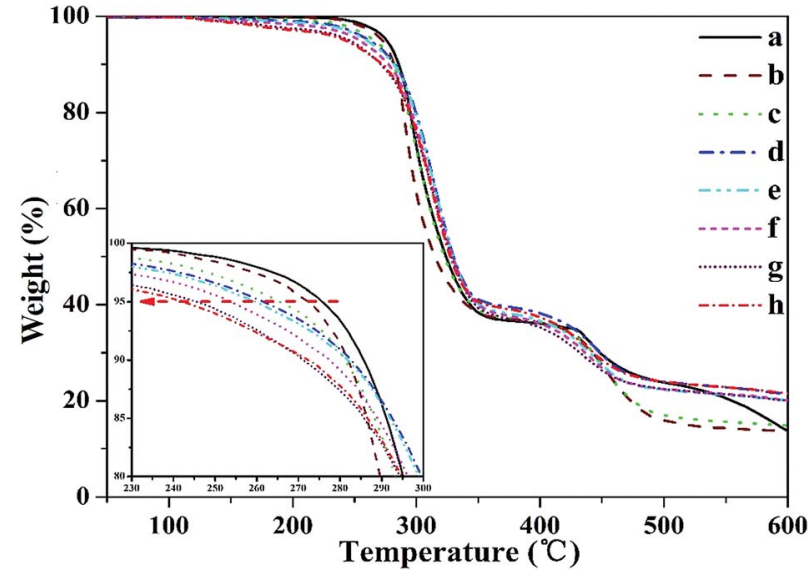

Fig. 6 TGA curves of PVC under different alkali treatment times (a-0 $\mathrm{h}$, b-1 h, c-3 h, d-5 h, e- 8 h, f-10 h, g-12 h, and h-13.5 h). b-1 h, 0.2407-0.005961) with those obtained through thermal pretreatment at 5 days (Table 1 , sample d-5 d, 0.3119-0.0054) showed that the content of conjugated polyene sequence structure of the former was lower than that of the latter; however, their internal allylic chlorine structures (Table 5, sample b- $1 \mathrm{~h}, 0.6$; Table 2, sample d-5 d, 0.7) and $T_{5 \%}$ values (Table 3, sample d-5 d, $275.0{ }^{\circ} \mathrm{C}$; Table 6, sample b-1 h, 272.4 $\left.{ }^{\circ} \mathrm{C}\right)$ were similar. These data indicated that the internal allylic chloride structure significantly affected the initial thermal stability of PVC.

Internal allylic chloride structures, which induced PVC degradation, played a crucial role in the initial thermal stability of the resin. These structures easily induced PVC degradation and led to relatively low thermal stability. Thus, dehydrochlorination was predominantly initiated at the internal allylic chloride structure sites and promoted degradation. 
Table 6 Thermal decomposition results of PVC as a function of alkali pretreatment times

\begin{tabular}{|c|c|c|c|c|c|c|c|c|}
\hline $\begin{array}{l}\text { Alkali pretreatment } \\
\text { time }(\mathrm{h})\end{array}$ & $a-0 h$ & $\mathrm{~b}-1 \mathrm{~h}$ & $c-3 \mathrm{~h}$ & $\mathrm{~d}-5 \mathrm{~h}$ & e-8 h & $f-10 h$ & $\mathrm{~g}-12 \mathrm{~h}$ & $\mathrm{~h}-13.5 \mathrm{~h}$ \\
\hline$T_{5 \%}\left({ }^{\circ} \mathrm{C}\right)$ & 274.8 & 272.4 & 265.6 & 261.7 & 259.8 & 253.7 & 271.2 & 242.4 \\
\hline
\end{tabular}

\section{Conclusion}

In this study, a series of PVC samples was prepared separately through thermal and alkali pretreatment to construct the CSM and the ASM, respectively. The FTIR analyses of the pretreated samples as a function of time eliminated the influence of the oxygen-containing groups in the PVC molecular chain. The UVVis and ${ }^{1} \mathrm{H}-\mathrm{NMR}$ results showed that the models were successfully constructed. These models demonstrated that the initial thermal stability of the PVC was minimally affected by the conjugated sequences structure but was greatly influenced by the internal allylic chloride structure. By comparing the ASM with the CSM, the dehydrochlorination reaction of PVC was found to be predominantly initiated at the internal allylic chlorine sites, thereby further promoting degradation.

\section{Acknowledgements}

We gratefully acknowledge financial support from the National Natural Science Foundation of China (No. 21464012), Program for Changjiang Scholars and Innovative Research Team in University (No. IRT_15R46), Funds for Distinguished Young Scientists of Xinjiang Bintuan (No. 2014CD001) and Scientific Research Program of Shihezi University (No. RCZX201407).

\section{References}

1 I. Boughattas, M. Ferry, V. Dauvois, C. Lamouroux, A. Dannoux-Papin, E. Leoni, E. Balanzat and S. Esnouf, Polym. Degrad. Stab., 2016, 126, 219-226.

2 I. Boughattas, E. Pellizzi, M. Ferry, V. Dauvois, C. Lamouroux, A. Dannoux-Papin, E. Leoni, E. Balanzat and S. Esnouf, Polym. Degrad. Stab., 2016, 126, 209-218.

3 C. D'Aquino, W. Balmant, R. Ribeiro, M. Munaro, J. Vargas and S. Amico, Polym. Test., 2012, 31, 638-644.

4 K. Deshmukh and G. M. Joshi, Polym. Test., 2014, 34, 211219.

5 A. Marongiu, T. Faravelli, G. Bozzano, M. Dente and E. Ranzi, J. Anal. Appl. Pyrolysis, 2003, 70, 519-553.

6 N. A. Mohamed, N. A. A. El-Ghany, M. M. Fahmy and M. H. Ahmed, Polym. Test., 2015, 44, 66-71.

7 B. Wu, S. Qi and X. Wang, Polym. Test., 2010, 29, 717-722. 8 T. T. Nagy, B. Iván, B. Turcsányi, T. Kelen and F. Tüdös, Polym. Bull., 1980, 3, 613-620.

9 Z. Huang, C. Feng, H. Guo and X. Huang, Polym. Chem., 2016, 7, 3034-3045.

10 Z. Huang, A. Ding, H. Guo, G. Lu and X. Huang, Sci. Rep., 2016, 6, 25508-25519.

11 M. Fisch and R. Bacaloglu, Plast., Rubber Compos., 1999, 28, 119-124.
12 W. H. Starnes, J. Polym. Sci., Part A: Polym. Chem., 2005, 43, 2451-2467.

13 A. A. Yassin and M. W. Sabaa, J. Macromol. Sci., Rev. Macromol. Chem., 1990, 30, 491-558.

14 W. Manzoor, S. Yousaf and Z. Ahmad, Polym. Degrad. Stab., 1996, 51, 295-299.

15 D. Braun, A. Michel and D. Sonderhof, Eur. Polym. J., 1981, 17, 49-56.

16 E. Buruiană, A. Airinei, G. Robilă and A. Caraculacu, Polym. Bull., 1980, 3, 267-271.

17 T. Hjertberg and E. M. Sörvik, Polymer, 1983, 24, 685-692.

18 W. Starnes, Prog. Polym. Sci., 2002, 27, 2133-2170.

19 B. Troitskii and L. Troitskaya, Eur. Polym. J., 1997, 33, 12891294.

20 A. Caraculacu and E. Bezdadea, J. Polym. Sci., Polym. Chem. Ed., 1977, 15, 611-620.

21 B. Iván, J. P. Kennedy, T. Kelen and F. Tüdos, J. Polym. Sci., Polym. Chem. Ed., 1981, 19, 679-685.

22 B. Iván, J. P. Kennedy, T. Kelen, F. Tüdõs, T. T. Nagy and B. Turcsányi, J. Polym. Sci., Polym. Chem. Ed., 1983, 21, 2177-2188.

23 B. Iván, F. Tüdős, O. Egyed and T. Kelen, Makromol. Chem., Rapid Commun., 1982, 3, 727-731.

24 M. K. Naqvi, J. Macromol. Sci., Rev. Macromol. Chem., 1987, 27, 559-592.

25 M. F. Llauro-Darricades, N. Bensemra, A. Guyot and R. Petiaud, Makromol. Chem., Macromol. Symp., 1989, 171184.

26 K. Abbås and E. Sörvik, J. Appl. Polym. Sci., 1976, 20, 23952406.

27 A. Holländer, H. Zimmermann and J. Behnisch, Eur. Polym. J., 1991, 27, 959-963.

28 G. Talamini and G. Pezzin, Makromol. Chem., 1960, 39, 2638.

29 V. Chytrý, B. Obereigner and D. Lim, Eur. Polym. J., 1969, 5, 379-388.

30 X. G. Zheng, L. H. Tang, N. Zhang, Q. H. Gao, C. F. Zhang and Z. B. Zhu, Energy Fuels, 2003, 17, 896-900.

31 R. Bacaloglu and M. Fisch, Polym. Degrad. Stab., 1994, 45, 315-324.

32 R. Bacaloglu and M. Fisch, Polym. Degrad. Stab., 1995, 47, 33-57.

33 J. Liu, Y. Lv, Z. Luo, H. Wang and Z. Wei, RSC Adv., 2016, 6, 31898-31905.

34 K. Patel, A. Velazquez, H. Calderon and G. Brown, J. Appl. Polym. Sci., 1992, 46, 179-187.

35 Z. Luo, H. Wang, K. Chen, J. Liu, C. Wu and Z. Wei, Int. J. Polym. Anal. Charact., 2015, 20, 240-249.

36 S. Ma, J. Lu and J. Gao, Energy Fuels, 2002, 16, 338-342. 
37 T. Nagy, B. Turcsányi, T. Kelen and F. Tüdős, React. Kinet. Catal. Lett., 1978, 8, 7-11.

38 C. Huggett and B. C. Levin, Fire Mater., 1987, 11, 131-142.

39 J. Michal, Fire Mater., 1976, 1, 57-62.

40 Q. Sun, X. Shi, Y. Lin, Z. He, W. Xiao and C. Cheng, J. China Univ. Min. Technol., 2007, 17, 242-245.

41 M. Darricades-Llauro, A. Michel, A. Guyot, H. Waton, R. Petiaud and Q. Pham, J. Macromol. Sci., Chem., 1986, 23, 221-269.
42 P. D'Antuono, E. Botek, B. Champagne, J. Wieme, M.-F. Reyniers and G. B. Marin, J. Phys. Chem. B, 2008, 112, 14804-14818.

43 J. Purmova, K. F. Pauwels, W. Van Zoelen, E. J. Vorenkamp, A. J. Schouten and M. L. Coote, Macromolecules, 2005, 38, 6352-6366.

44 V. Bărboiu, G. Robilǎ, E. Buruiană and A. Caraculacu, Makromol. Chem., 1982, 183, 2667-2674. 